\title{
User-centered virtual environment design for virtual rehabilitation
}

\author{
Cali M Fidopiastis ${ }^{1 *}$, Albert A Rizzo², Jannick P Rolland ${ }^{3}$
}

\begin{abstract}
Background: As physical and cognitive rehabilitation protocols utilizing virtual environments transition from single applications to comprehensive rehabilitation programs there is a need for a new design cycle methodology. Current human-computer interaction designs focus on usability without benchmarking technology within a user-inthe-loop design cycle. The field of virtual rehabilitation is unique in that determining the efficacy of this genre of computer-aided therapies requires prior knowledge of technology issues that may confound patient outcome measures. Benchmarking the technology (e.g., displays or data gloves) using healthy controls may provide a means of characterizing the "normal" performance range of the virtual rehabilitation system. This standard not only allows therapists to select appropriate technology for use with their patient populations, it also allows them to account for technology limitations when assessing treatment efficacy.
\end{abstract}

Methods: An overview of the proposed user-centered design cycle is given. Comparisons of two optical seethrough head-worn displays provide an example of benchmarking techniques. Benchmarks were obtained using a novel vision test capable of measuring a user's stereoacuity while wearing different types of head-worn displays. Results from healthy participants who performed both virtual and real-world versions of the stereoacuity test are discussed with respect to virtual rehabilitation design.

Results: The user-centered design cycle argues for benchmarking to precede virtual environment construction, especially for therapeutic applications. Results from real-world testing illustrate the general limitations in stereoacuity attained when viewing content using a head-worn display. Further, the stereoacuity vision benchmark test highlights differences in user performance when utilizing a similar style of head-worn display. These results support the need for including benchmarks as a means of better understanding user outcomes, especially for patient populations.

Conclusions: The stereoacuity testing confirms that without benchmarking in the design cycle poor user performance could be misconstrued as resulting from the participant's injury state. Thus, a user-centered design cycle that includes benchmarking for the different sensory modalities is recommended for accurate interpretation of the efficacy of the virtual environment based rehabilitation programs.

\section{Background}

Over the past 10 years, researchers have explored the use of virtual environments (VEs) as a rehabilitation tool [1-4]. Although studies have documented successful re-training and transfer of training while utilizing this paradigm [5,6], there are few studies that suggest methods of designing VEs that transition from specific applications of cognitive re-training to comprehensive rehabilitation training programs $[7,8]$. Given that most

\footnotetext{
* Correspondence: cfidopia@uab.edu

'School of Health Professions, University of Alabama-Birmingham,
} Birmingham, AL, USA
VE applications for cognitive retraining require customized applications [9], cost effectiveness is an initial design consideration [7]. However, there is some evidence that when designed following a user-centered design cycle, VE platforms can be validly and reliably applied across therapy scenarios [10].

"Good Fit" assessments are another suggested requirement of the virtual rehabilitation (VR) design cycle. The purpose of these assessments is to gauge how well the VE solution presents real world attributes in a more controlled, repeatable manner that will allow for comparable results over treatment effects [10]. This point

\section{Biomed Central}


raises an important issue: VE solutions for cognitive rehabilitation are mostly designed to capture data necessary to evaluate levels of cognitive function or transfer effects pre and post rehabilitation. As such, they are inherently guided by experimental design and scientific principles. This fact argues for standardized design methodologies when constructing VR environments, especially for applications that target persons with cognitive impairments. Lack of standardization leads to redundancy of VE applications and platforms; more importantly, it makes comparisons across research endeavors difficult [11].

International guidelines do exist for designing computer-based systems that are user-centered and iterative throughout the design lifecycle. Specifically, the International Standard ISO13407, the Human-Centered Design Process for Iterative Systems, outlines principles of human-centered design that account for user context, computer-system design, and environment of use within an iterative design cycle [12]. Usability evaluation, ease of use and utility, is a key component to the user-centered design methodology. The main goals of usability within the design cycle are to ensure system effectiveness, efficiency, safety, and utility [13]. VE based trainers for medical and military applications involving person without cognitive impairments have been successfully designed using the ISO 13407 framework [14,15]. However, satisfying the recommended guidelines is a subjective endeavor and determining valid usability testing for persons with impairments such as anterograde amnesia may require more medical community agreement.

Further, Stanney [16] contended that human sensory and motor physiology in general may prove to be limiting factors in some aspects of VE design. The HumanComputer Interaction (HCI) community has proposed varying general VE systems design approaches including those that focus on perceptual issues $[17,18]$, usability $[19,20]$, or combined perceptual and usability models $[21,22]$. Yet, there are several technological and computer graphics issues that lead to degraded perception in VEs that may confound VE rehabilitation assessments [23].

For example, a VE system may utilize a head-worn display (HWD). Microdisplays within HWDs typically limit the user's visual resolution acuity [23]. Further, HWD optical systems with a single imaging plane may also affect the natural accommodation and convergence mechanisms of the human visual system, thereby degrading depth cue information [24]. The resulting visual performance errors have the potential to distort experimental results, including those obtained from brain imaging.

When evaluating VE system design, separating the human component from the engineering component may prove difficult [25]. Melzer and Moffit [26] addressed this issue by applying user-centered methodologies to HWD design cycles. Figure 1 represents an example of a user performance model for HWDs adapted from Eggleston [27]. The user performance model outlines the interdependencies of HWD properties, computer graphics techniques, and their combined effects upon the user's perception of the VE. More specifically, the model illustrates how errors in the hardware (HWD optics and display) and software (Computer Graphics) impact the user's ability to correctly perceive the constructed VE space. The user also contributes his or her individual differences in perceptual abilities (e.g., spatial processing) to the overall error. Thus, the model must also include a user perception to image level twoway interaction as illustrated in Figure 1.

Following the user-centered design model, the HWD designer is not only responsible for usability from a user's perspective, but from the software perspective as well. Thus, HWD designers are concerned with limits in HWD parameters such as display resolution (image quality), field of view (information quantity), and contrast (light intensity changes) [28]. Just as with user-centered HWD design, user-centered VE design considers the standard limits of the human visual system (e.g., visual acuity, contrast modulation, and stereoacuity) as minimal user requirements for optimal viewing of the VE scene [29]. In contrast, some researchers suggest that visual errors may be caused more by the graphical techniques used to define the spatial layout of the VE $[30,31]$. Thus, even with a well designed and calibrated HWD, the VE may not support proper viewing conditions for successful task completion.

To circumvent these technology, graphics, and user issues, an interactive and iterative VE design cycle that includes sensory performance metrics for establishing baselines within a cognitive rehabilitation VE is proposed and diagramed in Figure 2. The design cycle integrates the requirements of the International Standard ISO13407, while including components of the more successful HCI VE design guidelines [22]. Sensory performance is measured before and during the design phase to ensure that the technology assembled is appropriate for the rehabilitation protocol. In addition, performance baseline metrics are obtainable. These metrics allow for the cross comparison of VE rehabilitation systems and the means to separate user performance from technology limitations during experimental analysis.

The VE rehabilitation system is not built until the component technology and graphical methods meet the task requirements for the rehabilitation protocol. A more important outcome of this methodology is that rehabilitation specialists can understand empirically the best VE system designs for providing effective treatment 


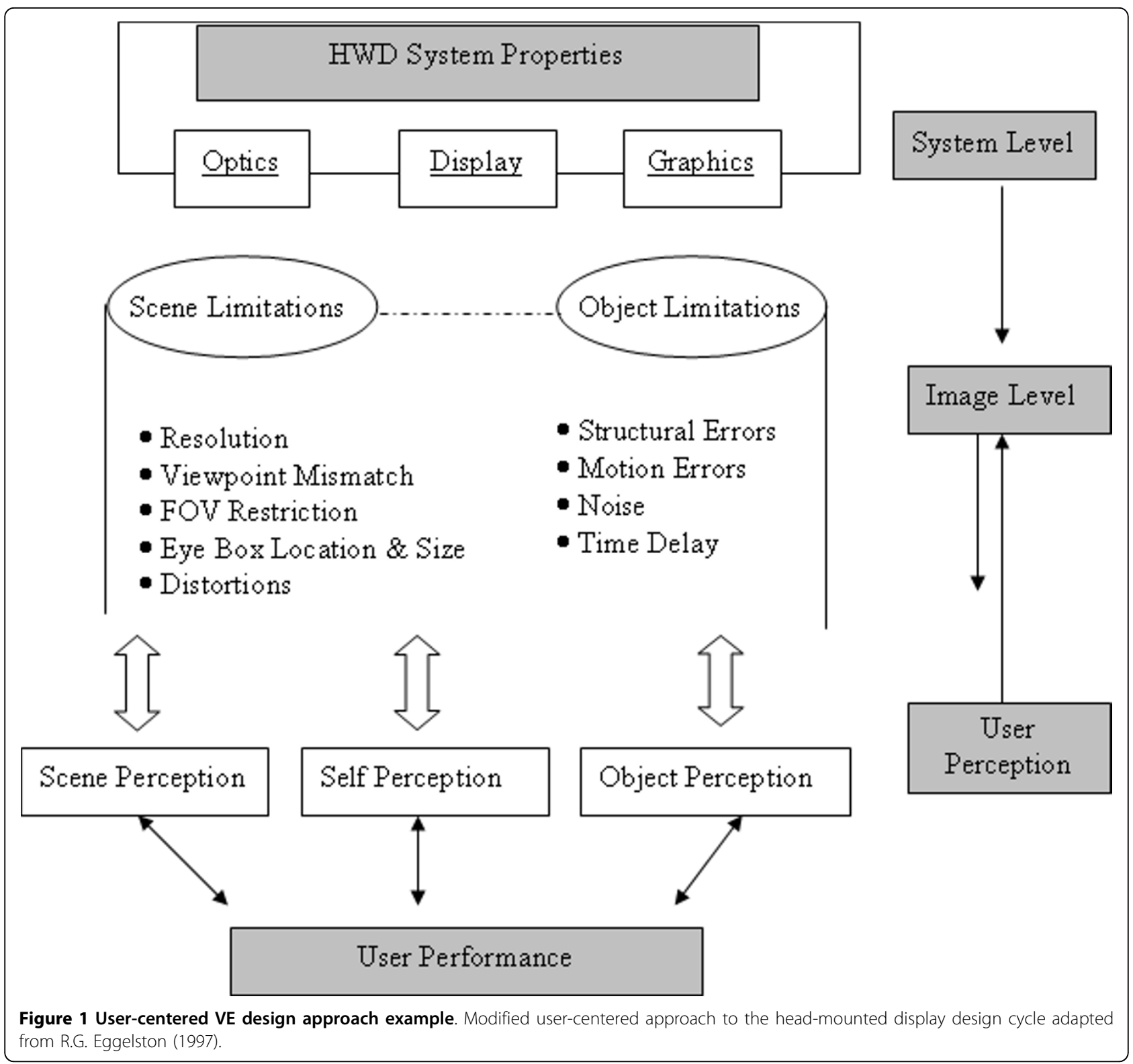

for persons experiencing cognitive impairments. Thus, the VE rehabilitation application is extendable to a successful, cost effective, and comprehensive rehabilitation program.

An ongoing impediment to VE system design is that usability assessments lack appropriate sensory tests (vision, auditory, smell, and touch) to provide accurate benchmarks for VE systems (i.e., technologies, computer graphics, and users). As a step toward narrowing this gap, we present modules of a vision test battery that quantifies key components of the human image processing system, namely resolution visual acuity and depth perception modules $[23,32,33]$. In this paper, we shall present results obtained with the stereoacuity module.
The test battery can be performed when considering different types of VE methods (e.g., augmented reality) as well as with varying types of display technologies (e.g., projectors, monitors or HWDs). The results of such a battery should provide basic and applied vision parameters for the total VE system, which will allow for appropriate benchmarks and performance evaluations that control for visual errors (e.g., distorted depth) within VE based cognitive rehabilitation applications.

\section{Methods}

\section{HWD technology}

The purpose for performing tasks while wearing a seethrough HWD is that real and virtual world objects are 


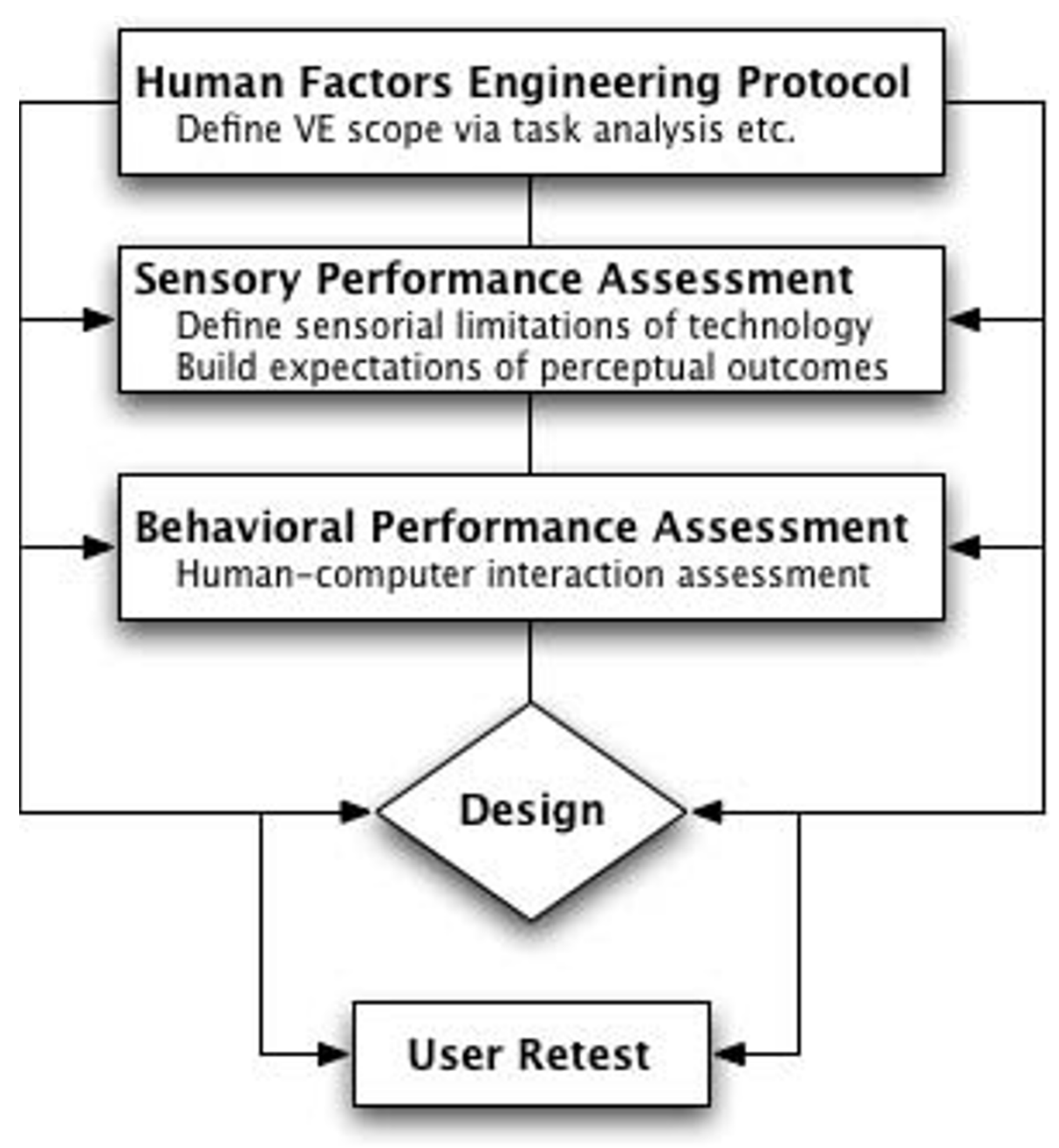

\section{Meet Requirements}

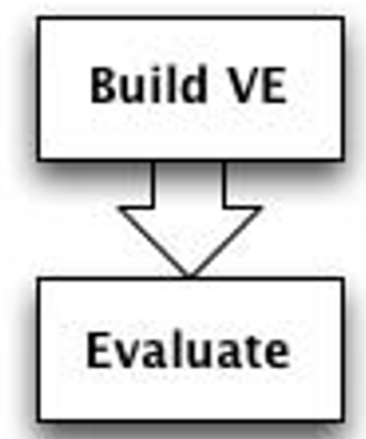

Figure 2 Proposed iterative VE design cycle. Proposed interactive iterative VE design cycle including sensory performance metrics for establishing baselines within a cognitive rehabilitation VE. 
combined to make up the task space. For example, some types of therapy may be best performed utilizing virtual components (e.g., stove burners) along with real world objects (e.g., dials for setting heat) instead of replicating the total rehabilitation setting in a solely virtual counter part. There are two choices for see-though displays and they are categorized based upon how they merge the real and virtual scene: optical see-through HWDs employ a semi-transparent mirror, while video-see through HWDs use a video camera (see [34] for a comprehensive review).

Optical see-through HWDs are typically associated with augmented reality tasks whereby the virtual world is overlaid onto real objects [35]. Figure 3 pictures two optical see-through displays, first and second generation prototype head-worn projection displays (HWPDs) whose optics were developed in the ODALab at the College of Optics and Photonics at the University of Central Florida [36]. Because the original stereoacuity assessment was conducted using the bench prototype of the first generation HWPD (HWPD-1), the HWPD-1 and HWPD-2 (second generation) were used in the experiment.

\section{HWPD parameters}

Table 1 Technology specifications for HWPD-1 and HWPD-2 used in experiments. Table 1 provides the technical specifications for the HWDs worn during the stereoacuity testing. Information such as display type, field-of-view (FOV), interpupillary eye distance (IPD) range, and resolution are important parameters of the
HWD that determine the users' visual performance. For example, resolution as imposed by the microdisplay can be estimated by measuring the average subtense of a single pixel in either the horizontal or vertical dimension after being magnified by the optics. This resolution value can be computed from the horizontal or vertical resolution given in pixels and the FOV for that dimension. The approximated resolution can then be compared to that of the human visual system. The resolution visual acuity of the human eye is accepted as 1 arc minute or 20/20 [37]. Comparatively, the measured resolution visual acuity for users wearing the HWPD-1 is 4.1 arc minutes ( 20/80) and 2.73 arc minutes $(\sim 20 / 60)$ for the HWPD-2 [38]. Thus, the user wearing the HWPD-2 should be able to see better object detail than the person wearing the HWPD-1; however, factors such as display brightness as determined by the display type (e.g., liquid crystal or organic light-emitting) and graphical content can play a role in detail visibility.

Most often, researchers do not report the optical depth plane of the HWD; however, this parameter is critical to understanding visual perception issues in VEs. The virtual image created by the HWD is usually magnified and presented at a fixed distance from the observer, usually between $500 \mathrm{~mm}$ and infinity $[39,40]$. This fixed distance is based upon the optics of the HWD system and may result in conflicts between the accommodation and the convergence mechanisms of the eye. Although multi-focal plane HWDs have been proposed [41], they are not available on today's HWD market. As a result, the focus distance of HWDs does not

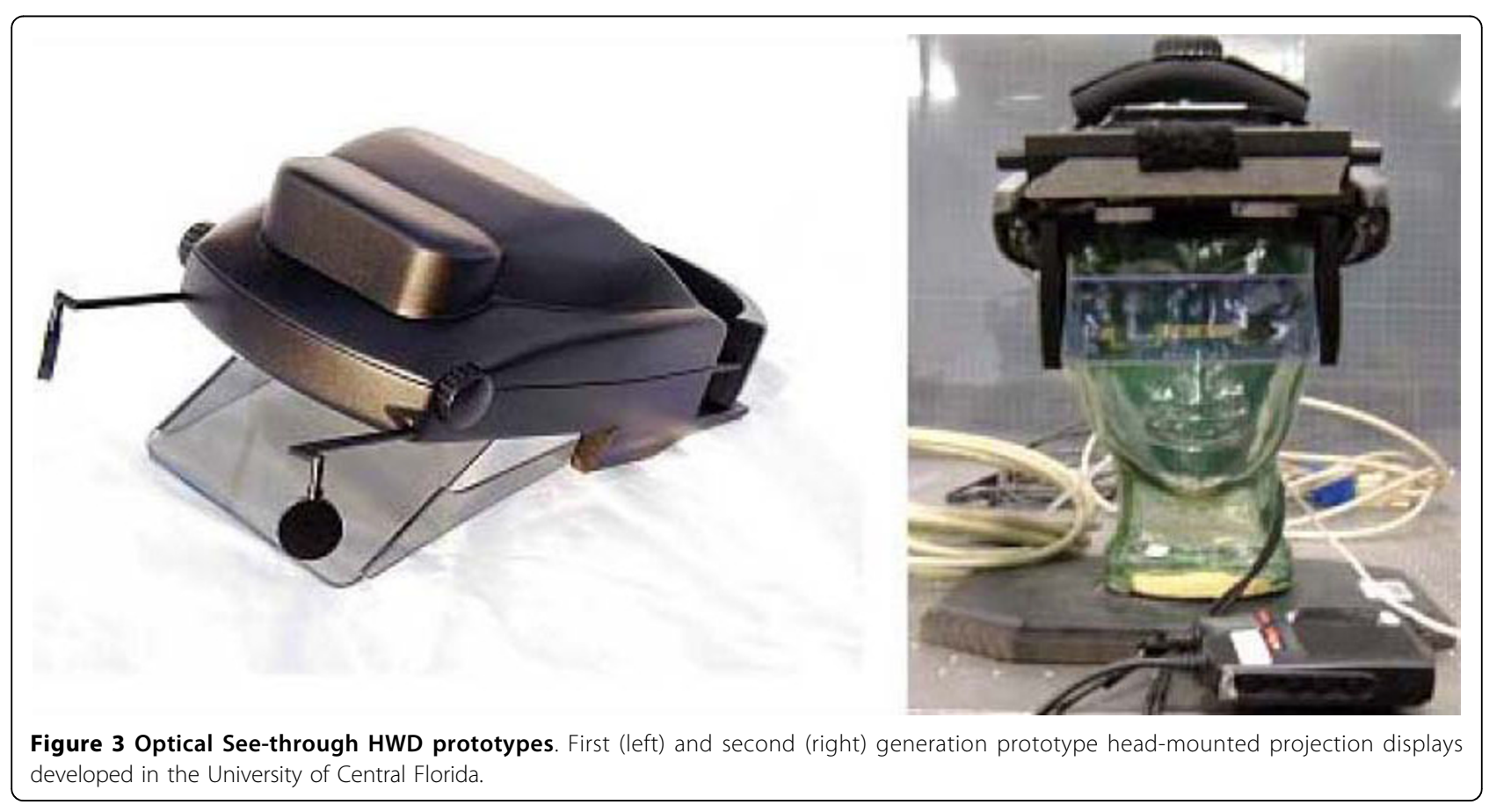


Table 1 HWD technology specifications. HWPD-1 and HWPD-2 specifications used in the experiments.

\begin{tabular}{|c|c|c|c|c|c|c|c|c|}
\hline \multirow[b]{2}{*}{ HMD Type } & \multirow[b]{2}{*}{ Display Type } & \multicolumn{2}{|c|}{ FOV (Degree) } & \multicolumn{2}{|c|}{ Resolution (Pixels) } & \multirow[b]{2}{*}{$\begin{array}{c}\text { Display Size } \\
\text { mm }\end{array}$} & \multirow[b]{2}{*}{$\begin{array}{c}\text { Focus Plane } \\
\mathrm{mm}\end{array}$} & \multirow[b]{2}{*}{ IPD mm } \\
\hline & & $\mathrm{H}$ & V & $\mathrm{H}$ & v & & & \\
\hline HMPD-1 & LCD & 41 & 31 & 640 & 480 & $27 \times 20$ & Infinity & $55-75$ \\
\hline 800 & & 40 & 31 & & & & 800 & \\
\hline 1500 & & 41 & 31 & & & & 1500 & \\
\hline 3000 & & 42 & 32 & & & & 3000 & \\
\hline \multicolumn{9}{|l|}{ HMPD-2 } \\
\hline 800 & OLED & 33 & 25 & 800 & 600 & $12 \times 9 \mathrm{~mm}$ & 800 & $55-72$ \\
\hline 1500 & & 34 & 26 & & & & 1500 & \\
\hline 3000 & & 34 & 26 & & & & 3000 & \\
\hline Canon & LCD & 57 & 37 & 640 & 480 & & 2000 & 63 \\
\hline VR6 & LCD & 48 & 36 & 640 & 480 & $1.3 \times 2.59$ & 914 & $52-74$ \\
\hline
\end{tabular}

a Horizontal and Vertical

b Depth of the optical plane (i.e., depth at which the image is rendered)

dynamically change as does the human eye to allow for focus on near or far objects. Because both optical seethrough displays were custom built, we could adjust the focus planes for both HWPDs to present the virtual image at different viewing depths from the observer. The importance of this adjustment is that the virtual image and the rendered image are collocated on the optical plane, thus eliminating the mismatch between accommodation and convergence mechanisms of the observers' eyes.

The optics for HWPD-1 was optimized for infinity viewing (i.e., viewing distances $>6 \mathrm{~m}$ ) by design, thus the optical depth plane for this display is typically set to display images at infinity. However, the optics of this display also allow for adjustments to the optical depth plane, and thus allow for viewing distances of 800, 1500, or 3000 $\mathrm{mm}$ with only a slight decrement in resolution. Comparatively, the optics for HWPD-2 were optimized to technically operate at viewing depths of 800,1500 , and 3000 $\mathrm{mm}$ Because of this inherent design specification, the adjustments of the optical depth plane for HWPD-2 do not imply a compromise in image resolution. In the forthcoming experiments, we assessed the participants' stereoacuity at 800,1500 , and $3000 \mathrm{~mm}$ to confirm the depth presentation capabilities of each display.

It is important to note that the mismatch between the accommodation and the convergence mechanisms of the human eye is also accentuated by computer graphics techniques. More specifically, computer graphics render objects under infinity viewing conditions because the virtual cameras are considered as single fixed points or eyepoints [42]. How computer graphics techniques interact with technology constraints to impact user performance is another reason that establishing perceptual baselines are important to include in studies that involve learning or retraining.

\section{Stereoacuity benchmark test design}

Wann and Mon-Williams [17] argued that VEs should support "salient perceptual criteria" such as binocular vision that allow for the appropriate perception of spatial layout, which in turn supports naturalistic interaction (p. 835). Their contention that VEs design must center upon the perceptual-motor capabilities of the user is an important design criteria for extending VEs to rehabilitation scenarios. Rehabilitation scenarios involving Activities of Daily Living (ADLs) may necessitate a level of complexity and realism beyond simple reaching tasks and manipulating virtual objects to traversing a virtual grocery store and handling real objects. In addition, correct spatial locations of objects within a virtual space may be necessary to support transfer of training to the home.

Visual performance testing may be difficult since when viewing a mixed reality scene the visual abilities of the user are dependent upon the sensory characteristics of the virtual and real objects (e.g., brightness and contrast) as well as the layout of the VE space [43]. Further, the human eye is an optical system that is functionally limited much like the HWD in such parameters as resolution. Given that the HWD parameters are designed with respect to limitations of the human eye, clinical vision tests which elucidate the functional limitations of the human eye are applicable to testing visual performance when the human eye is coupled with an HWD. Thus, we chose vision tests associated with established methodologies and real world correlates.

Clinical tests for stereoacuity can be divided into two categories, real-depth tests and projected-depth tests. The Howard-Dolman two-peg test is a classic example of a real-depth test whereby a real test object is moved in and out of the plane of one or more target objects [44]. The amount of difference in alignment between 
the two objects determines stereoacuity sensitivity. Stereograms, which present left and right eye perspective views of an image to the viewer, are examples of projected-depth tests. Although projected-depth tests are capable of eliminating most secondary depth cues, which diminish the accuracy of real-depth tests, the presentation of these tests are not reliable in VEs [45]. The modified virtual Howard-Dolman task (V-HD task) developed by [32] and later improved upon by [33] qualifies in general as a projected-depth test. At this time, the assessment provides the best metric for measuring stereoacuity with regard to VE system assessment.

Figure 4 displays each of the stereoacuity assessments performed during the experiment. Prior to testing, participants were screened using the Titmus Stereo Test, a standard projected-depth test, to confirm that their stereoacuity was at least 40 arc seconds. These tests are shown in Figure $4 \mathrm{a}$ and $4 \mathrm{~b}$, respectively. Further, the modified Howard-Dolman peg test using the Howard Dolman apparatus was performed before and after VE testing to monitor changes in visual performance over the course of the experiment.

The stimuli presented during the V-HD task are pictured in Figure 4c. The V-HD task controls for the familiar size cues by presenting generic objects, an octahedron and a cylinder), which have no real world correlation [32]. Thus, there is no expectation of size when simultaneously viewing both objects. However, aspects of the graphics such as lighting may provide a weak depth cue. Rolland et al [33] adjusted for conflicts between accommodation and convergence mechanisms of the human eye by placing the microdisplay with regard to the optics such that the monocular optical images matched the location at which the $3 \mathrm{D}$ virtual objects were rendered.

\section{Participants}

This research was approved by the Institutional Review Board (IRB) of the University of Central Florida. Ten healthy male participants were randomly placed in either the HWPD-1 (mean age $=29.8$, SD $=5.26$ ) or HWPD-2 (mean age $=30.6, \mathrm{SD}=5.36$ ) viewing group. The Titmus Stereo Test confirmed that participants' stereoacuity was at least 40 arc seconds prior to the start of the experiment. As well, each participant was either corrected for or had 20/20 vision. If needed, glasses or contacts were worn during each part of the experiment.

\section{Procedure}

In this experiment, the participant performed the virtual Howard-Dolman (V-HD) task for two trials at a viewing distance of 800,1500 or $3,000 \mathrm{~mm}$. This viewing distance was randomly selected and each participant repeated the experiment on separate days until the stereoacuity assessment was performed at each distance. Before and after each virtual trial, the participant performed the modified Howard-Dolman task at the same viewing distance for that testing session to monitor possible changes in the participant's stereoacuity due to the VE exposure.

When performing the V-HD task, the HWD was adjusted for each person based upon comfort as well as IPD for each viewing distance. The virtual cylinder (target) was rendered at the chosen focus plane $(800,1500$, or $3000 \mathrm{~mm}$ ) and kept stationary. The virtual octahedron was randomly placed to the right or to the left of the cylinder, as well as in front of or behind the target object. The participant moved the octahedron using a dial so that its center was aligned with the center of the cylinder.

The response variables for this assessment were: 1) percent correct for whether the octahedron appeared in front of or behind the target; 2) the absolute constant error defined as the magnitude of the offset between the aligned objects; and, 3) the variable error or measure of dispersion about the participant's mean error score. The equivalence disparity metric $(\eta)$, a measure of stereoacuity, was calculated from the absolute constant error and the variable error metrics $[46,38]$. The percent

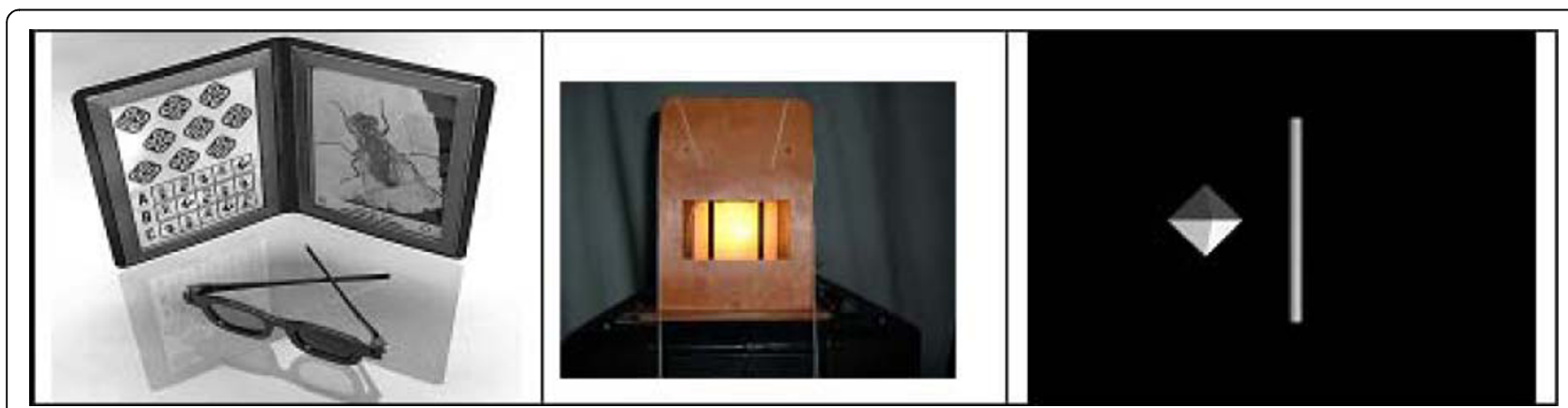

Figure 4 Depth perception tests and examples of stimuli. Titmus stereo test (left), Modified Howard-Dolman Apparatus (middle), Virtual Howard-Dolman stimuli (right). 
correct for front and back responses with the stereoacuity values are reported and discussed for each HWD tested.

\section{Results}

Stereoacuity calculated for HWPD-1 and HWPD-2

Figure 5 and 6 show the overall mean stereoacuity values attained at each viewing distance, for each task, and each HWPD. The bottom reference line at 20 arc seconds represents typical stereoacuity values for the Howard-Dolman task. In Figure 5, the reference line at 240 arc seconds represents the predicted stereoacuity based on the size of a single pixel as determined by HWPD-1 parameters, which was previously given as 4.1 arc minutes. The predicted stereoacuity for HWPD-2 is 156 arc seconds or 2.73 arc minutes and appears as the reference line in Figure 6.

\section{Percent correct front and back for HWPD-1 and HWPD-2}

The mean percent correct for responding whether the octahedron appeared in front of or behind the static cylinder prior to alignment is shown in Figure 7 and 8 for HWPD-1 and HWPD-2, respectively. This measure represents a 2 alternative-forced-choice (AFC) response where any score 75 percent and above meets the detection threshold. This threshold is indicated by dotted lines in both figures.

\section{Discussion}

One aim of this study was to introduce a stereoacuity test capable of benchmarking HWDs. Stereoacuity of each HWD was evaluated given their respective display parameters utilizing a user-in-the-loop methodology. The results showed that there was no significant difference between groups when performing the Howard-Dolman task at any viewing distance. Thus, subsequent differences found between the groups may be attributed to the type of HWPD worn while performing the virtual Howard-Dolman task.

As figures 5 and 6 show, the participants' performance was better than the predicted stereoacuity based on the pixel size resolution of each display, 240 and 156 arc seconds, respectively. Participants wearing HWPD-1 performed more variably at the $800 \mathrm{~mm}$ viewing distance; however, as the distance was adjusted toward the optimized optical plane, participants' performance improved significantly, $\left(\mathrm{M}_{\mathrm{V}-\mathrm{HD} 800}=186.70\right.$ arc sec, $\mathrm{SD}$ $=92.10$ arc sec; $M_{\mathrm{V}-\mathrm{HD} 1500}=133.52$ arc sec, $\mathrm{SD}=34.6$; $\left.\mathrm{M}_{\mathrm{V}-\mathrm{HD} 3000}=41.91 \mathrm{arc} \mathrm{sec}, \mathrm{SD}=7.18\right)$. This result is expected since the HWPD-1 is designed to perform

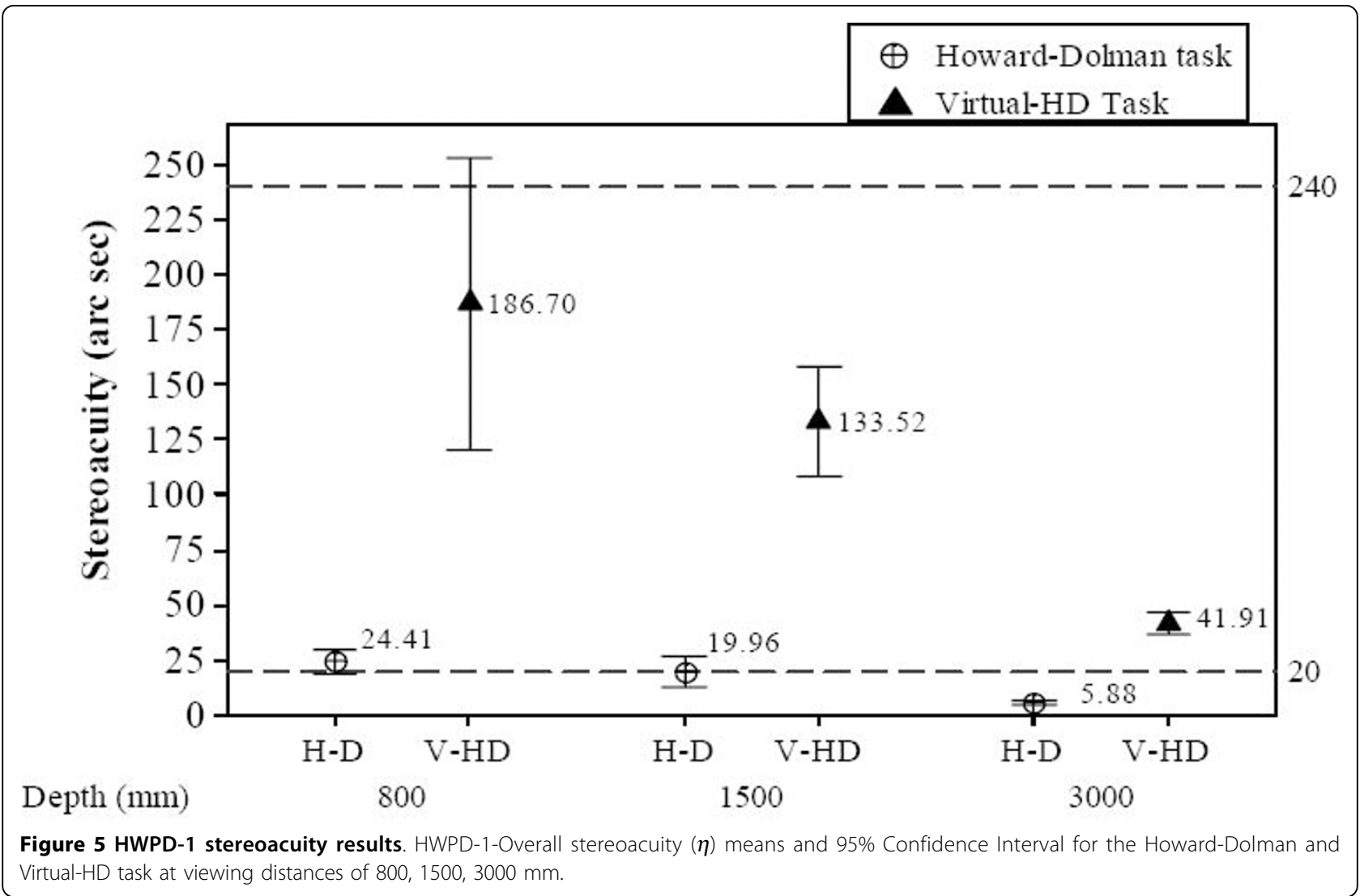



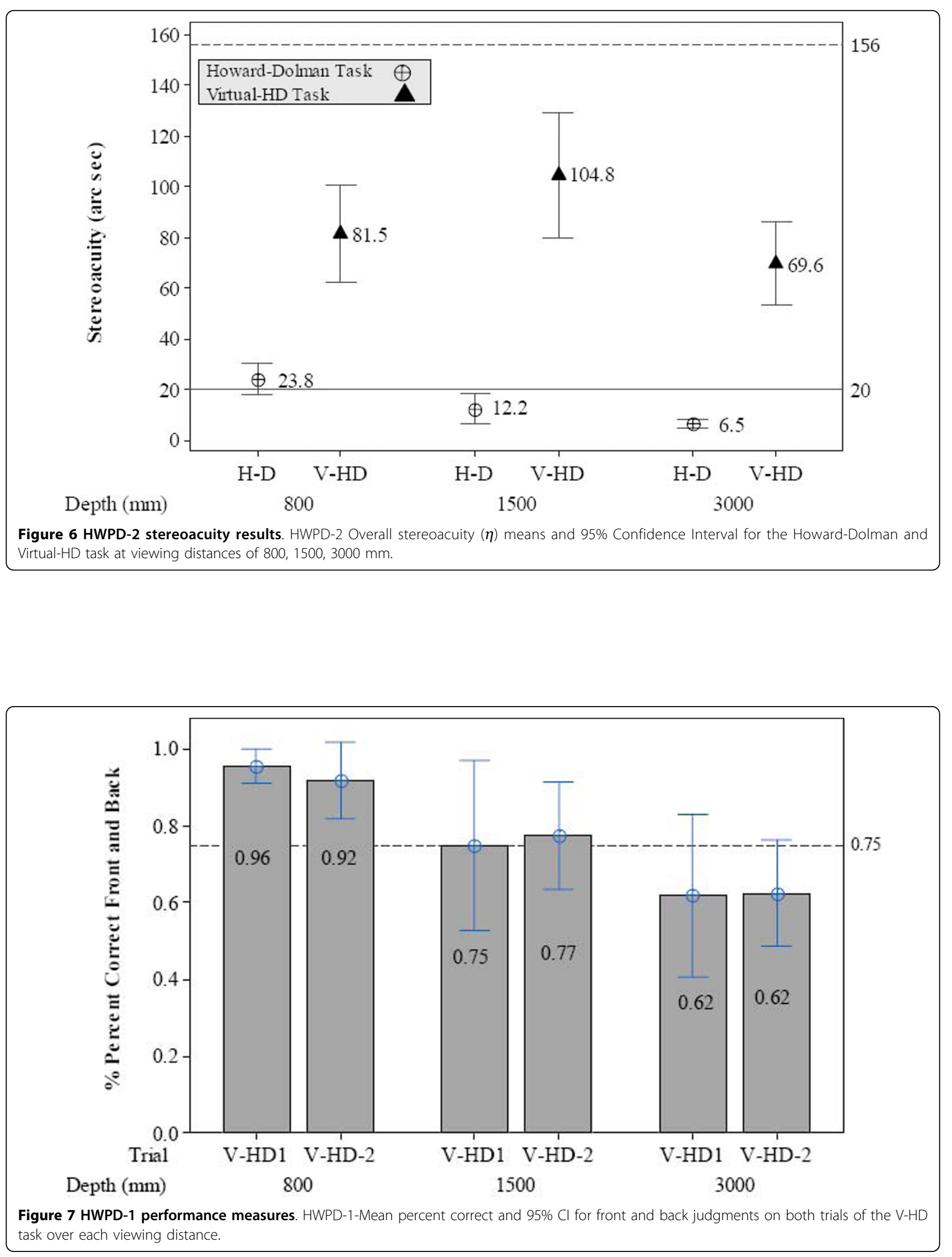


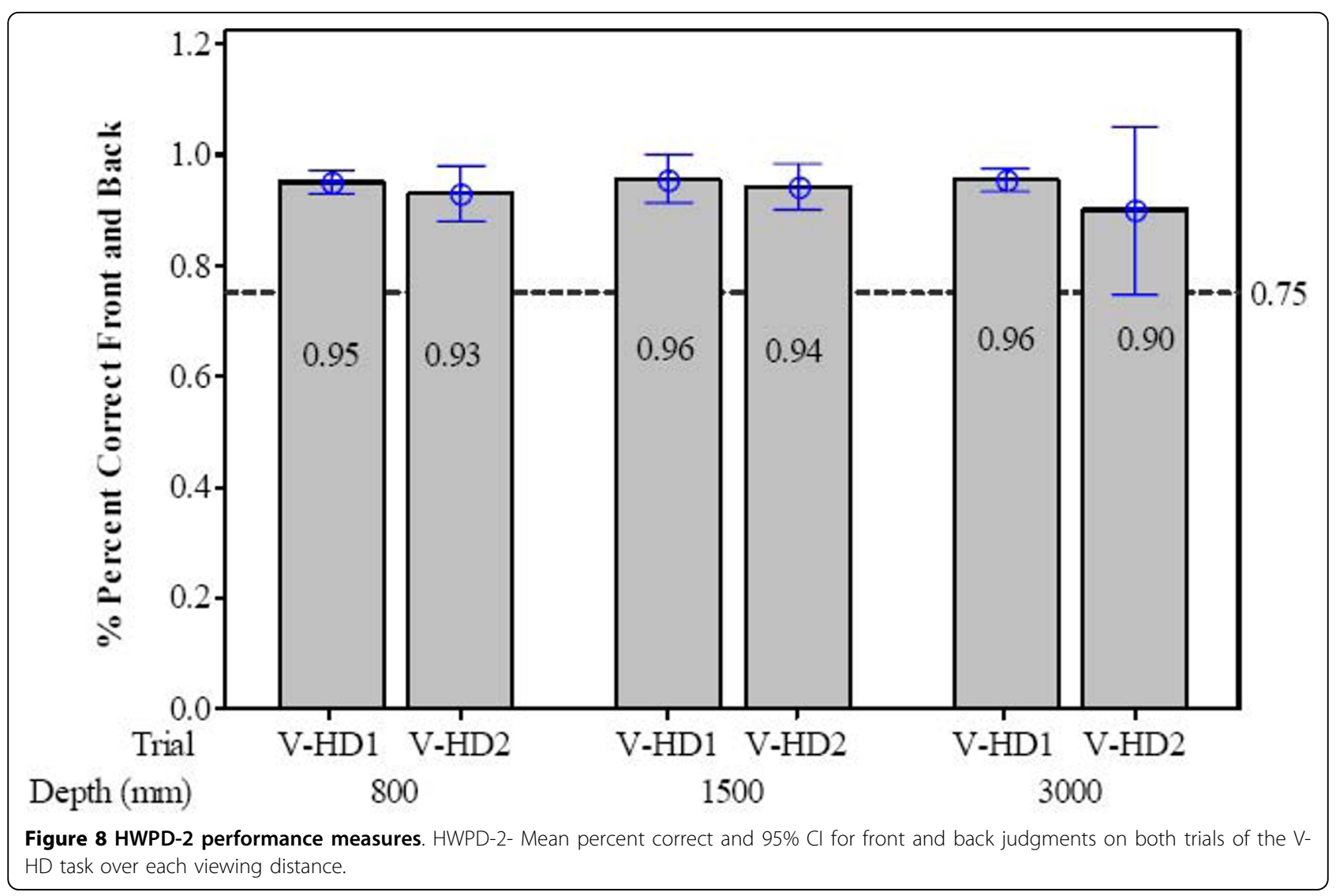

optimally at infinity viewing conditions. In contrast, stereoacuity for persons wearing HWPD-2, which is optically optimized across each viewing distance, does not change significantly with viewing distance, $\mathrm{M}_{\mathrm{V}-\mathrm{HD} 800}$ $=81.46, \mathrm{SD}=27.01, \mathrm{M}_{\mathrm{V}-\mathrm{HD} 1500}=104.80, \mathrm{SD}=34.70$, $\mathrm{M}_{\mathrm{V}-\mathrm{HD} 3000}=69.6, \mathrm{SD}=23.05$.

These results suggest that HWPD-1 may not be the best candidate HWD for performing tasks requiring good stereoacuity in personal space (i.e., within arms reach). However, HWPD-1 does attain stereoacuity levels closer to those attained under natural viewing conditions when the optical depth plane is set to infinity or the setting for which it was optimized. The stereoacuity levels for HWPD-2 are not maximized for any one viewing distance. It is known that stereoacuity improves with improved binocular visual acuity $[47,48]$. Thus, although HWPD-2 provides better binocular visual acuity than HWPD-1, this advantage is diminished for the $3000 \mathrm{~mm}$ condition because of the requirement of optimizing the optics across the additional optical plane settings. This finding points to the benefit of designing HWDs to target a specific field of use for which visual task performance must be optimized.

It should also be noted that the stereoacuity scores obtained when wearing either HWPD are lower than the predicted real-world stereoacuity values for the same levels of visual acuity attainable by each HWPD. Real world stereoacuity predictions for a Snellen score of 20/ 80 range from 178 to 200 arc seconds, which matches the visual acuity attainable by HWPD-1. For a Snellen score of 20/60, which corresponds to HWPD-2, predicted stereoacuity values range from 160 to 200 arc seconds $[47,49]$. Figures 5 and 6 show that HWPD- 1 and HWPD- 2 match or best these predicted values. This improvement in stereoacuity is attributed to antialiasing techniques which improve the visibility of edges of the rendered objects. This result suggests interdependence between image resolution of the rendered virtual objects and computer graphics techniques that should be accounted for when assessing VE systems for rehabilitation therapies.

Figures 7 and 8 display the percent correct responses for determining whether the octahedron appeared in front of or behind the cylinder before aligning the objects while performing the task wearing HWPD-1 or HWPD-2. While wearing HWPD-1, the participants were able to perform above threshold for the 800 and the $1500 \mathrm{~mm}$ viewing distances; however, they failed to meet threshold for the $3000 \mathrm{~mm}$ viewing distance. As Figure 7 shows, participants performed similarly for 
both repetitions of the task at the $3000 \mathrm{~mm}$ viewing distance, $\mathrm{M}_{\mathrm{V}-\mathrm{HD} 1}=.62, \mathrm{SD}=.17$ and $\mathrm{M}_{\mathrm{V}-\mathrm{HD} 2}=.62, \mathrm{SD}=$ .11. Thus, the task was difficult for participants to perform even with prior exposure to the test stimuli at that distance.

In comparison, Figure 8 shows that participants did not respond below $90 \%$ correct when evaluating front and back initial positions of the octahedron relative to the fixed cylinder while performing the task wearing HWPD-2. There are several properties of HWPD-2 that may contribute to better performance. First, the display type is an OLED, Organic Light Emitting Diode, which provides more brightness and contrast than the standard LCD, Liquid Crystal Display, of HWPD-1 (Cakmakci, 2006). As well, the increased resolution acuity of the HWPD-2 also lends to an improved resolvable depth (i. e., the range of distances in front of and behind the optical plane where a single pixel size is just resolvable) over HWPD-1.

These results suggest that HWD characteristics reduce stereoacuity for persons with unimpaired vision. This reduction is quantifiable using the virtual Howard-Dolman task. Rehabilitation specialists can compare these benchmarks to assess the appropriateness of different HWDs for use in their VR protocols. For example, HWPD-1 may not be an appropriate choice for close distance therapeutic tasks (e.g., dropping a letter in a virtual mailbox) as the stereoacuity limitations may augment the patient's propensity for errors. These design decisions can occur early in the design process, thus reducing time and cost while potentially improving the overall effectiveness of the VR therapy.

\section{Conclusions}

HCI guidelines are important in VE systems design; however, their goals and methods may change to accommodate for the sensory and perceptual deficits of persons with cognitive impairment. For example, persons with anterograde amnesia may not remember their VE exposure even 10 minutes post training. Questionnaires or verbal report methods may not be appropriate for this population; however, procedural tasks that support motor memory may prove useful. Thus, VE design for VR must match research concerns with the comfort, the safety, and the acceptability of the user. Research and safety constraints may be best satisfied with baseline metrics representing both clinical and unimpaired populations.

Task characteristics also determine the level of realism, immersion, and overall detail necessary to achieve the desired training effect. For example, pain management and exposure therapies using VE technologies do not demand high levels of graphical detail or realism to achieve positive behavioral outcomes [50,51]. However, rehabilitation protocols for assisting persons with spatial neglect should ensure that distortions of shape and size constancy, as well as depth errors caused by the VE technology are controlled or at least understood prior to therapy and experimentation.

As more technology providers (e.g., haptic devices) follow, convergence between the engineering and user requirements will simplify the VE design decision making. However, benchmarks addressing user sensory capabilities across separated and integrated technologies are necessary when evaluating user performance, especially when evaluating persons with brain injuries. These benchmarks are critical to evaluating negative adaptation effects as well as positive transfer of training.

More specifically, we argue that current user-centered design approaches are too high level to support the empirical nature of VR. When building a specific rehabilitation application, it is appropriate to introduce sensory performance assessments after the initial task analysis is performed. However, general sensory baselines attained via a user-in-the-loop methodology are the responsibility of the device manufacturer. The goal of the therapist is not to determine the individual limits of the technology from a user perspective, but to define the integrative effects of technology as they assist or detract from user performance in a rehabilitation scenario.

\section{Acknowledgements}

This research was supported by the Office of Naval Research Grants N000140210261 and N00014-02-1-0927, as well as a Link Foundation Fellowship awarded to the first author.

\section{Author details}

${ }^{1}$ School of Health Professions, University of Alabama-Birmingham, Birmingham, AL, USA. ${ }^{2}$ Integrated Media Systems Center, University of Southern California, Los Angeles, California, USA. ${ }^{3}$ Institute of Optics, University of Rochester, Rochester, NY, USA.

\section{Authors' contributions}

AAR provided the foundational concepts for VR system design, including benchmarking. CMF conceived of the additions to the design cycle, participated in the design of the stereoacuity test, implemented the study, performed the analyses, and drafted the manuscript. JPR conceived of the study along with the stereoacuity test and the experimental design. JPR also assisted with data analyses and the editing of the manuscript. All authors read and approved the final manuscript.

\section{Competing interests}

The authors declare that they have no competing interests.

Received: 3 August 2008

Accepted: 19 February 2010 Published: 19 February 2010

\section{References}

1. Rizzo AA, Buckwalter JG: Virtual reality and cognitive assessment and rehabilitation: the state of the art. Virtual Reality in Neuro-Physiology Amsterdam, Netherlands, IOS PressRiva G 1997, 123-146.

2. Rizzo AA, Kim GJ: A SWOT Analysis of the field of virtual rehabilitation and therapy. Presence 2005, 14(2):119-146. 
3. Schultheis MT, Rizzo AA: The application of virtual reality technology for rehabilitation. Rehab Psychol 2001, 46(3):296-311.

4. Rose FD, Brooks BM, Rizzo AA: Virtual reality in brain damage rehabilitation: Review. CyberPsychol \& Behav 2005, 8(3):241-262.

5. Rose FD, Attree EA, Brooks BM, Parslow DM, Penn PR, Ambihapahan N: Training in Virtual Environments: transfer to real world tasks and equivalence to real task training. Ergonomics 2000, 43:494-511.

6. Fidopiastis CM, Stapleton CB, Whiteside JD, Hughes CE, Fiore SM, Martin GM, Rolland JP, Smith EM: Human experience modeler: Contextdriven cognitive retraining to facilitate transfer of learning. CyberPsychol \& Behav 2006, 9(2):183-187.

7. Rizzo AA, Buckwalter JG, Zaag Van der C: Virtual environment applications in clinical Neuropsychology. Handbook of virtual environments: Design, implementation, and application Mahwah, NJ: Lawrence ErlbaumStanney K 2002, 1027-1063.

8. Rizzo AA, Kim GJ, Yeh SC, Thiebaux M, Hwang J, Buckwalter G: Development of a benchmarking scenario for testing 3D user interface devices and interaction methods. Proceedings of the 11th International Conference on Human Computer Interaction. Las Vegas, Nevada 2005.

9. Weiss PL, Rand D, Katz N, Kizony R: Video capture virtual reality as a flexible and effective rehabilitation tool. J of NeuroEng and Rehab 2004, $1-1$.

10. Rizzo AA, Bowerly T, Buckwalter JG, Klimchuk D, Mitura R, Parsons TD: A virtual reality scenario for all seasons: the virtual classroom. CNS Spectrum 2006, 11(1):35-44.

11. Moreira da Costa RM, Vidal de Carvalho LA: Virtual reality in cognitive retraining. Proceedings of IWALT 2000, the International Workshop on Advanced Learning Technology Palmerston North, New Zealand, 4-6 December, Los Alamitos, CA: IEEE Computer Society 2000, 221-224.

12. ISO: ISO 13407- Human-centered design processes for interactive systems. International Organization for Standardization 1999.

13. Preece J, Rogers Y, Sharp H: Interaction design: Beyond human-computer interaction. New York, NY: John Wiley \& Sons 2002.

14. Stedmon AW, Stone RJ: Re-viewing reality: Human factors of synthetic training environments. Int Jof Human-Computer Studies 2001, 55:675-698.

15. Riva G: Applications of virtual environments in medicine. Meth of Inform in Med 2003, 42:524-534.

16. Stanney KM: Realizing the full potential of virtual reality: human factors issues that could stand in the way. Proceedings of IEEE Virtual Reality Annual International Symposium '95, Research Triangle Park, North Carolina March 11-15, 1995 28-32.

17. Wann JP, Mon-Williams M: What does virtual reality NEED?: Human factors issues in the design of three-dimensional computer environments. Intl J of Human-Computer Studies 1996, 44:829-847.

18. Fencott C: Towards a design methodology for virtual environments. Poster presented at the User Centered Design and Implementation Workshop September 30, York England 1999http://www.cs.york.ac.uk/hci/ kings_manor_workshops/UCDIVE/.

19. Kaur K, Maiden N, Sutcliffe A: Interacting with virtual environments: an evaluation of a model of interaction. Interacting with Computers 1999, 11:403-426.

20. Bowman DA, Gabbard JL, Hix D: A survey of usability evaluation in virtual environments: Classification and comparison of methods. Presence 2000, 11(4):404-424.

21. Stanney KM, Mourant RR, Kennedy RS: Human factors issues in virtual environments: A review of the literature. Presence 1998, 7(4):327-351.

22. Stanney KM, Mollaghasemi M, Leah R, Breau R, Graeber DA: Usability engineering of virtual environments (Ves): identifying multiple criteria that drive effective VE system design. Intl I of Human-Computer Studies 2003, 588:447-481.

23. Fidopiastis CM, Furhman C, Meyer C, Rolland JP: Methodology for the iterative evaluation of prototype head-mounted displays in virtual environments: visual acuity metrics. Presence 2005, 14(5):550-562.

24. Wann JP, Rushton S, Mon-Williams M: Natural problems for stereoscopic depth perception in virtual environments. Vision Research 1995, 35(19):2731-2736.

25. Kalawsky RS: The science of virtual reality and virtual environments: a technical, scientific and engineering reference on virtual environments. Wokingham, England: Addison-Wesley 1993.

26. Melzer E, Moffit K: Head Mounted Displays: Designing for the User New York, NY: McGraw-Hill 1997.
27. Eggelston RG: User-centered design in the trenches: Head-mounted display system design and user performance. Head Mounted Displays: Designing for the user New York, NY: McGraw-HillMelzer JE, Moffit K 1997, 17-54.

28. Task HL: HMD Image source, optics, and the visual interface. Head Mounted Displays: Designing for the user New York, NY: McGraw-HillMelzer JE, Moffit K 1997, 55-82.

29. May JG, Badcock DR: Vision and Virtual Reality. Handbook of Virtual Environments Technology Lawrence Erlbaum Associates, IncStanney KM 2002, 29-63.

30. Cutting JE, Vishton PM: Perceiving layout and knowing distances: The interaction, relative potency, and contextual use of different information about depth. Perception of space and motion San Diego, CA: Academic PressEpstein W, Rogers S 1995, 69-117.

31. Ellis SR: Seeing. Pictorial communication in virtual and real environment Washington, DC: Taylor \& FrancisEllis SR, Kaiser MK, Grunwald AJ 1993, 419-424.

32. Rolland JP, Gibson W, Ariely D: Toward quantifying depth and size perception in virtual environments. Presence 1995, 4:24-49.

33. Rolland JP, Meyer C, Arthur K, Rinalducci E: Methods of adjustments versus method of constant stimuli in the quantification of accuracy and precision of rendered depth in head-mounted displays. Presence 2002, 11(6):610-625.

34. Rolland J, Fuchs H: Optical versus video see-through head-mounted displays in medical visualization. Presence 2001, 9(3):287-309.

35. Davis L, Rolland JP, Hamza-Lup F, Ha Y: Enabling a continuum of virtual environment experiences. IEEE Computer Graphics and Applications 2003, $2-4$

36. Rolland JP, Hua H: Head-mounted display systems. Encyclopedia of Optical Engineering New York, NY: Marcel DekkerDriggers RG 2005, 1-13.

37. Goss DA, West RW: Introduction to the optics of the eye Boston, MA: Butterworth-Heinemann 2001

38. Fidopiastis CM: User-centered virtual environment assessment and design for cognitive rehabilitation applications. PhD dissertation, University of Central Florida, Orlando, FL. Dissertations \& Theses database 2006, (Publication No. AAT 3233649).

39. Cakmakci O, Rolland J: Head-worn displays: a review. IEEE/OSA Journal of Display Technology 2006, 2(3):199-216.

40. Velger M: Helmet-mounted Displays and Sights Boston, MA: Artech House, Inc 1998.

41. Rolland JP, Kreuger MW, Goon A: Multifocal planes head-mounted displays. Applied Optics 2000, 39(19):3209-3215.

42. Robinett W, Rolland JP: A computational model for the stereoscopic optics of a head-mounted display. Presence 1992, 1(1):45-62.

43. Bullimore MA, Howarth PA, Fulton J: Assessment of visual performance. Evaluation of human work: a practical ergonomics methodology Nottingham, U.K:: Taylor \& FrancisWilson JR, Corlett EN 1995, 804-839.

44. Reading RW: Binocular Vision: Foundations and Applications Boston, MA: Butterworth Publishers 1983.

45. Wong BP, Woods RL, Peli E: Stereoacuity at distance and near. Opt and Vis Sci 2002, 79(12):771-778.

46. Ogle KN: Visual optics and the optical space sense. The Eye New York, NY: Academic PressDavidson H 1962, 4:271-324.

47. Donzis PB, Rapazzo JA, Burde RM, Gordon M: Effect of binocular variations of Snellen's visual acuity on Titmus stereoacuity. Arch Opthalmol 1983, 101:930-932.

48. Goodwin RT, Romano PE: Stereoacuity degradation by experimental and real monocular and binocular amblyopia. Invest Ophthal Visual Science 1985, 26:917-923.

49. Menon V, Bansal A, Prakash P: Randot stereoacuity at various binocular combinations of Snellen acuity. Indian J of Opthal 1997, 45(3):169-171.

50. Rothbaum $\mathrm{BO}$, Hodges LF: The use of virtual reality exposure in the treatment of anxiety disorders. Behav Mod 1999, 23(4):507-525.

51. Hoffman HG, Patterson DR, Carrougher GJ, Sharar S: The effectiveness of virtual reality based pain control with multiple treatments. Clin J of Pain 2001, 17(3):229-235.

doi:10.1186/1743-0003-7-11

Cite this article as: Fidopiastis et al:: User-centered virtual environment design for virtual rehabilitation. Journal of NeuroEngineering and Rehabilitation 2010 7:11. 